\title{
LISTADO SISTEMÁTICO DE LAS AVES DEL ESTERO BOCA NEGRA, MÉXICO, REGISTRADO EN ABRIL DE 2004.
}

\section{SYSTEMATIC LIST OF THE BIRDS FROM BOCA NEGRA ESTUARY, MEXICO, REGISTERED IN APRIL 2004.}

\author{
Fabio Germán Cupul-Magaña ${ }^{1}$
}

\section{Resumen}

Se presenta una lista sistemática de aves en el estero Boca Negra, Jalisco, México. Se visitó la zona del 3 al 9 de Abril de 2004. Se determinaron 35 especies pertenecientes a nueve órdenes y 19 familias. Del total, 19 fueron acuáticas y 16 terrestres. El estero soporta parte de la actividad reproductiva regional de las aves; por lo que requiere de protección, en vista de las presiones derivadas del desarrollo urbano.

Palabras clave: estero, Boca Negra, aves, México.

\begin{abstract}
The systematic list of birds observed at Boca Negra estuary, Jalisco, Mexico, is presented. The area was visited from April 3 to 9, 2004. Thirty five species, belonging to nine orders and 19 families were observed. Of these, 19 were water birds and 16 were land birds. The estuary is the most important place in this region that supports bird reproductive activity. For this reason it should be protected considering urban development derived pressures.
\end{abstract}

Key words: estuary, Boca Negra, birds, Mexico.

\section{Introducción}

El estero Boca Negra posee una superficie de 0.15 $\mathrm{km}^{2}$ y se encuentra al norte de la ciudad de Puerto Vallarta, Jalisco, México, entre los $20^{\circ} 39^{\prime}-20^{\circ} 42^{\prime} \mathrm{N}$ y $\operatorname{los} 105^{\circ} 15^{\prime}-105^{\circ} 17^{\prime} \mathrm{W}$ (Figura 1). Es un pequeño humedal costero rodeado de vegetación de manglar, con dominancia del mangle blanco Laguncularia racemosa (L.) Gaertner F., y cuyo volumen de agua se incrementa por las precipitaciones pluviales y escurrimientos durante la temporada de lluvias: JunioOctubre (Cupul-Magaña et al., 2002). Está ubicado dentro de una zona climática semicálida subhúmeda fresca, donde la temperatura y la precipitación pluvial promedio anual oscila entre $\operatorname{los} 26^{\circ} \mathrm{C}$ a $28^{\circ} \mathrm{C}$ y $\operatorname{los}$ $930.8 \mathrm{~mm}$ a $1668.0 \mathrm{~mm}$, respectivamente (García, 1981).

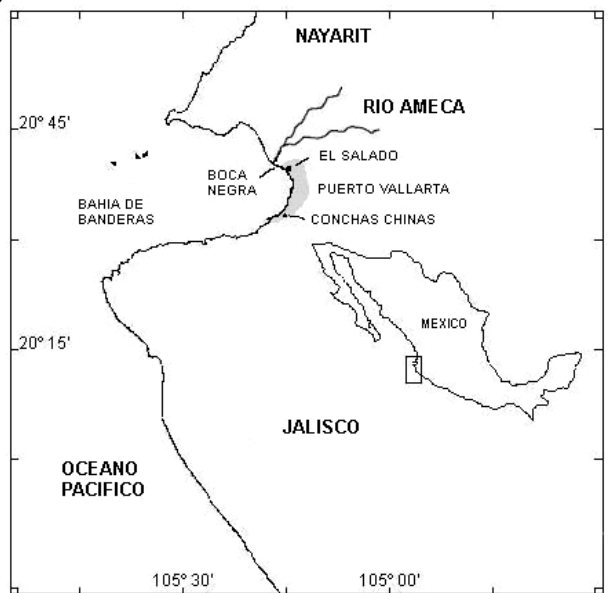

Figura 1.- Localización geográfica del estero Boca Negra, Jalisco, México.
Hasta hace poco tiempo, la importancia ecológica del estero Boca Negra como área de reproducción de cocodrilo de río (Crocodylus acutus Cuvier, 1807) y de aves acuáticas fue puesta en evidencia (CupulMagaña, 2000; Cupul-Magaña et al., 2002). Actualmente la zona experimenta reducciones en sus áreas naturales costeras por el uso del suelo para el desarrollo y crecimiento de la industria turística de playa y de bienes raíces, con lo que la permanencia de estos hábitats representativos se ve comprometida (Cupul-Magaña, 2000).

El objetivo es presentar un listado sistemático de las aves presentes en el estero Boca Negra a lo largo de una semana y durante el inicio de la temporada reproductiva, el mismo que se espera sea de utilidad para elaborar y reforzar la propuesta de protección legal del área.

\section{Materiales y Métodos}

El estudio se realizó del 3 al 9 de Abril de 2004. El estero se recorrió en una embarcación de aluminio a lo largo de sus canales $(800 \mathrm{~m})$. Los recorridos se realizaron entre las 08:00 y las 10:00 h y se acumuló un total de $14 \mathrm{~h}$ de observación. Las observaciones, tanto dentro del manglar como en el espejo de agua, se realizaron con la ayuda de binoculares 8x. La identificación de las aves fue en base a las guías de Peterson \& Chalif (1989) y Howell \& Webb (1995).

El arreglo taxonómico presentado para las especies fue el propuesto por la American Ornithologists' Union (1998). Además, se presenta su tipo de 
presencia en la región (visitante o residente) basada en el trabajo de Howell \& Webb (1995).

\section{Resultados y Discusión}

Se determinaron 35 especies pertenecientes a nueve órdenes y 19 familias. Del total, 19 fueron acuáticas $(54.28 \%)$ y 16 terrestres (45.72\%). Asimismo, 27 especies se consideran residentes y ocho como visitantes (Tabla 1).

A excepción de Larus atricilla (Linnaeus), Chlidonias niger (Linnaeus) y Stelgidopteryx serripennis (Audubon) que sobrevolaban a baja altura sobre el espejo de agua, el resto de las especies fueron observadas entre o sobre el manglar y en el espejo de agua. Además, dos especies se reportan como endémicas (Tabla 1) para el Occidente de México (Howell \& Webb, 1995).

Un resultado interesante fue la observación de 13 especies (11 acuáticas y dos terrestres) de aves anidando sobre la vegetación de manglar (Tabla 1). Por lo que, independientemente de que Boca Negra sea mucho más pequeño en extensión y en diversidad avifaunística que esteros vecinos como El Salado (1.4 $\mathrm{km}^{2}$ ) o El Quelele $\left(1 \mathrm{~km}^{2}\right)$, su valor como área de anidación es destacable, ya que en los dos cuerpos costeros mencionados con anterioridad, sólo se ha observado y documentado al menos a una especie de ave acuática en reproducción (Cupul-Magaña, 1999, 2003).

La significancia del estero Boca Negra como hábitat importante para las aves residentes, visitantes y anidantes, radica probablemente en que éste representa un sitio favorable para que permanezcan al contar con recursos alimenticios para explotar eficientemente, así como de protección en contra de la depredación (Custer et al., 1996; Carmona et al., 2003).

\section{Recomendaciones}

La presencia de un número importante de especies anidantes en un espacio tan reducido y con presiones por parte del desarrollo humano (Cupul-Magaña, 2004), ponen de manifiesto al estero Boca Negra como un sitio prioritario para la conservación dentro de la región costera de Puerto Vallarta, México.

\section{Literatura citada}

American Ornithologists' Union. 1998. Check-list of North American birds. Allen Press. Lawrence, Kansas, U. S. A.

Carmona R., Brabata G., Cuéllar-Brito A. \& González-Peralta A. 2003. Observaciones recientes de aves en el oasis de La Purísima, Baja California Sur, México. Anales del Instituto de Biología, Universidad Nacional Autónoma de México, Serie Zoología. 74(1): 43-52.

Cupul-Magaña F.G. 1999. La laguna El Quelele, Nayarit, México, como hábitat de aves acuáticas. Ciencia y Mar (México). 3(8): 21-28.
Tabla 1.- Listado sistemático de las especies de aves registradas en el estero Boca Negra, México, en abril de 2004. $\mathrm{A}=$ acuático, $\mathrm{T}=$ terrestre, $\mathrm{R}=$ residente, $\mathrm{V}=$ visitante, $\mathrm{An}=$ anidando, $\mathrm{E}=$ endémica de México.

\begin{tabular}{|c|c|c|c|}
\hline Lista Sistemática & 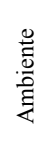 & 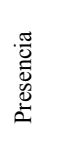 & 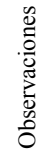 \\
\hline Podilymbus podiceps (Linnaeus) & $\mathrm{A}$ & $\mathrm{R}$ & \\
\hline Phalacrocorax brasilianus (Gmelin) & $\mathrm{A}$ & $\mathrm{R}$ & An \\
\hline Anhinga anhinga (Linnaeus) & $\mathrm{A}$ & $\mathrm{R}$ & An \\
\hline Ardea herodias (Linnaeus) & $\mathrm{A}$ & $\mathrm{V}$ & \\
\hline Ardea alba (Linnaeus) & A & $\mathrm{R}$ & An \\
\hline Egretta thula (Molina) & $\mathrm{A}$ & $\mathrm{R}$ & An \\
\hline Egretta caerulea (Linnaeus) & $\mathrm{A}$ & $\mathrm{R}$ & An \\
\hline Egretta tricolor (Müller) & $\mathrm{A}$ & $\mathrm{R}$ & An \\
\hline Bubulcus ibis (Linnaeus) & $\mathrm{A}$ & $\mathrm{R}$ & $\mathrm{An}$ \\
\hline Butorides virescens (Linnaeus) & $\mathrm{A}$ & $\mathrm{R}$ & An \\
\hline Nyctanassa violacea (Linnaeus) & $\mathrm{A}$ & $\mathrm{R}$ & $\mathrm{An}$ \\
\hline Cochlearius cochlearius (Linnaeus) & $\mathrm{A}$ & $\mathrm{R}$ & An \\
\hline Eudocimus albus (Linnaeus) & $\mathrm{A}$ & $\mathrm{R}$ & An \\
\hline Ajaia ajaja (Linnaeus) & $\mathrm{A}$ & $\mathrm{V}$ & \\
\hline Coragyps atratus (Bechstein) & $\mathrm{T}$ & $\mathrm{R}$ & \\
\hline Gallinula chloropus (Linnaeus) & $\mathrm{A}$ & $\mathrm{R}$ & \\
\hline Actitis macularia (Linnaeus) & $\mathrm{A}$ & $\mathrm{V}$ & \\
\hline Larus atricilla (Linnaeus) & $\mathrm{A}$ & $\mathrm{V}$ & \\
\hline Chlidonias niger (Linnaeus) & $\mathrm{A}$ & $\mathrm{V}$ & \\
\hline Geotrygon montana (Linnaeus) & $\mathrm{T}$ & $\mathrm{R}$ & \\
\hline Chloroceryle americana (Gmelin) & $\mathrm{A}$ & $\mathrm{R}$ & \\
\hline Melanerpes chrysogenys (Vigors) & $\mathrm{T}$ & $\begin{array}{c}R \\
(E)\end{array}$ & \\
\hline Pitangus sulphuratus (Linnaeus) & $\mathrm{T}$ & $\mathrm{R}$ & \\
\hline Myiozetetes similis (Spix) & $\mathrm{T}$ & $\mathrm{R}$ & An \\
\hline Tyrannus melancholicus (Vieillot) & $\mathrm{T}$ & $\mathrm{R}$ & \\
\hline Cyanocorax sanblasianus (Lafresnaye) & $\mathrm{T}$ & $\begin{array}{c}\mathrm{R} \\
(\mathrm{E})\end{array}$ & \\
\hline Stelgidopteryx serripennis (Audubon) & $\mathrm{T}$ & $\mathrm{R}$ & \\
\hline Polioptila caerulea (Linnaeus) & $\mathrm{T}$ & $\mathrm{V}$ & \\
\hline Dendroica petechia (Linnaeus) & $\mathrm{T}$ & $\mathrm{R}$ & \\
\hline Setophaga ruticilla (Linnaeus) & $\mathrm{T}$ & $\mathrm{V}$ & \\
\hline Seiurus noveboracensis (Gmelin) & $\mathrm{T}$ & $\mathrm{V}$ & \\
\hline Saltator coerulescens (Vieillot) & $\mathrm{T}$ & $\mathrm{R}$ & \\
\hline Quiscalus mexicanus (Gmelin) & $\mathrm{T}$ & $\mathrm{R}$ & $\mathrm{An}$ \\
\hline Icterus pustulatus (Wagler) & $\mathrm{T}$ & $\mathrm{R}$ & \\
\hline Cacicus melanicterus (Bonaparte) & $\mathrm{T}$ & $\mathrm{R}$ & \\
\hline
\end{tabular}

2000. Aves acuáticas del estero El Salado, Puerto Vallarta, Jalisco. Huitzil (México). 1: 3-7.

2003. Reproducción avanzada de Nyctanassa violacea (Ardeidae) en el estero El Salado, Jalisco, México (junio a julio de 2001). Ciencia y Mar (México). 8(21): 43-49.

2004. Observaciones sobre la reproducción de tres especies de ardeidos en el estero Boca Negra, Jalisco, México. Huitzil (México). 5(1): 7-11. 
Rubio-Delgado A., Reyes-

Juárez A. \& Hernández-Hurtado H. 2002. Sondeo poblacional de Crocodylus acutus en el estero Boca Negra, Jalisco. Ciencia y Mar (México). 6(16): 45-49.

Custer T.W., Hines R.K. \& Custer C.M. 1996. Nest initiation and clutch size of great blue herons on the Mississippi River in relation to the 1993 flood. Condor. 98: 181-188.

García E. 1981. Modificaciones al sistema de clasificación climática de Köppen (para adaptarlo a las condiciones de la República Mexicana). UNAM. Instituto de Geografía. México.

Howell S.N.G. \& Webb S. 1995. A guide to the birds of Mexico and Nothern Central America. Oxford University Press. Oxford.

Peterson R.T. \& Chalif E.L. 1989. Aves de México: guía de campo. Editorial Diana. México.

\footnotetext{
${ }^{1}$ Departamento de Ciencias, Centro Universitario de la Costa, Universidad de Guadalajara. Av. Universidad de Guadalajara \#203, Delegación Ixtapa, C.P. 48280, Puerto Vallarta, Jalisco, México. E-mail:

fcupul@hotmail.com
} 